\title{
EP-87
}

\section{Robotic living donor hepatectomy: Is it the future?}

\author{
Christina DELIMPALTA*
}

Department of General Surgery, Norfolk and Norwich University Hospital, Norwich, UK

Introduction: Donor liver hepatectomy has always been associated with significant risks and complications affect up to $1 / 3$ of patients. The vast majority are related to large incisions, severe postoperative pain, lung atelectasis, extensive intrabdominal adhesions as well as intraoperative blood loss and biliary injuries. The advent of robotic surgery can improve outcomes through minimally invasive approaches and use of superb technological instruments, but its use for donor hepatectomy is still in infancy. This review summarises its current state and challenges.

Methods: Review of published literature.

Results: Robotic liver surgery offers significant advantages: small access wounds, stable magnified 3-D intraabdominal views, multidimensional instrument articulation. For liver donor resection this allows close individual biliary and vascular dissection, improved haemostasis by ligating vascular pedicles precisely, parenchymal transection facilitated by better views and reduced blood loss. Even in the challenging right donor hepatectomy, a fourth robotic arm allows for identification of accessory veins and venous reconstruction if necessary. Published studies show longer operative time for the robotic population but comparable intraoperative blood loss and similar incidence of early allograft dysfunction, vascular complications (hepatic artery thrombosis, portal vein thrombosis or stenosis), and 1-year liver regeneration and liver function tests.

Conclusions: Robotic hepatectomy has the potential to revolutionize living donor liver surgery and to minimize major complications and their associated morbidity. However it is still limited to few highly expert centers and requires extensive operative experience in robotic and liver resections. As exposure to minimally invasive techniques grows, it could become the mainstay of practice in the next decade. 